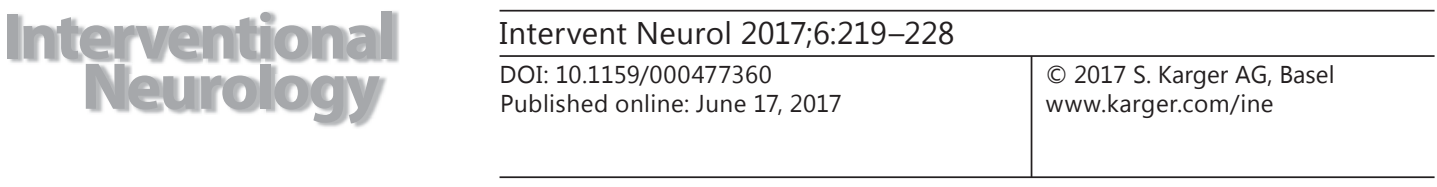

\title{
Optional Endovascular Therapy of Dissecting Posterior Cerebral Artery Aneurysm
}

\author{
Mohamed Wael Osman ${ }^{a} \quad K$ rzysztof Kadziolka ${ }^{b} \quad$ Laurent Peirot $^{b}$ \\ ${ }^{a}$ Neurology Department, Al-Azhar University, Cairo, Egypt; ${ }^{b}$ Intervention Neuroradiology \\ Department, CHU Reims, Reims, France
}

\section{Keywords}

Dissecting posterior cerebral artery aneurysm · Endovascular therapy

\begin{abstract}
Background: Posterior cerebral artery aneurysms are uncommon, with an occurrence rate of less than $1 \%$ of intracranial aneurysms. They have various shapes, including saccular and fusiform. Dissecting aneurysms may occur in distal posterior cerebral artery and they may affect the whole artery. Endovascular therapy is considered as a safe method of treatment and there are different techniques for endovascular therapy. Summary: Posterior cerebral artery aneurysms are uncommon. Endovascular therapy is considered as a safe method of treatment and there are different techniques for endovascular therapy. We present here three cases collected from Maison Blanche Hospital (Intervention Neuroradiology Department, CHU Reims, France) during 2011-2012; they were females, at a young age and the affected side was on the right. The first case was affected at the P2-P3 segment, the aneurysm was fusiform in shape and she presented with ischemic stroke, while the second and third cases were affected at the P2 segment, the aneurysms being saccular in shape; one of them presented with subarachnoid hemorrhage with a history of migraine and the other patient presented with ischemic stroke. All of them had no history of trauma, hypertension or other diseases. One patient was treated by coiling and sacrificing the parent artery, the second patient was treated with stent-assisted coils, and the third one was treated by coiling without sacrificing the parent artery.




\begin{tabular}{l|l}
\hline DOI: $10.1159 / 000477360$ & $\begin{array}{l}\text { C } 2017 \text { S. Karger AG, Basel } \\
\text { www.karger.com/ine }\end{array}$ \\
\hline
\end{tabular}

Wael Osman et al.: Optional Endovascular Therapy of Dissecting Posterior Cerebral Artery Aneurysm

\section{Introduction}

Posterior cerebral artery (PCA) aneurysms are uncommon, with an occurrence rate of less than $1 \%$ of intracranial aneurysms $[1,2]$. They have various shapes, including saccular and fusiform. Dissecting aneurysms may occur in the distal PCA and they may affect the whole artery $[3,4]$. The junction of the PCA and the posterior communicating artery is the demarcation point between the proximal P1 segment and the distal P2-P4 segments [5-8]. Dissecting aneurysms remain without detectable cause and can be posttraumatic or spontaneous. The surgical treatment of PCA aneurysm is difficult. Endovascular therapy by sacrificing the PCA after balloon occlusion test or using stent-assisted coiling to reconstruct the artery without sacrificing the PCA, and coiling without sacrificing are effective and safe. We present 3 cases of different ages, with dissection of the PCA using different techniques. Informed consent was obtained from all patients.

\section{Case 1}

A 19-year-old female patient, cigarette smoker, taking the contraceptive pill, presented with paresthesia of the left hand and face, with disturbance of her memory and attention, no visual field defect, and no motor system or cranial nerve affection. The patient sought medical advice. Brain imaging was done and showed right thalamic infarction. One year later, the patient again complained of second ischemic stroke, right thalamic and occipital region infarction, fever, and vomiting followed by headache (right frontal). She was not referred, had no history of joint affection, epistaxis, phlebitis or skin lesion. General examination was normal, while neurological examination showed hypothesia of her left face and hand, right upper quadrantonopia, and motor system 4/5 weakness of the left side of pyramidal distribution. Deep tendon reflexes were equal on both sides, superficial reflex plantar flexion was bilateral, and MRI and MRA were performed. There was no evidence of aneurysms or other detectable causes. Laboratory tests including homocystine were normal; the patient was recommended for digital subtraction angiography (DSA) which detected a fusiform dissecting aneurysm of the right PCA that needed endovascular therapy. The operation was done without neurological complications or residual neck (Fig. 1).

\section{Technique}

The operation was done under general anesthesia. Bifemoral puncture was performed using two 6-Fr Chaperon catheters at the level of the right and left femoral arteries, selective catheterization of the right internal carotid artery, followed by antero-posterior and lateral view. Selective catheterization of the left vertebral artery was done, followed by the antero-posterior, lateral and 3D view cerebral angiography. The results of angiography showed the presence of a fusiform dissection aneurysm in the right PCA at the P2-P3 segment, measuring $12 \mathrm{~mm}$ in length and 4.5-6 $\mathrm{mm}$ in height. At first, we accomplished the occlusion test with the balloon of remodeling Hyperglide $3 \times 10$, positioned at the upstream of entrance of the fusiform aneurysm, and injection of the right vertebral artery and the right carotid artery was done. This occlusion test allowed assessment of the collateral circulation. We confirmed the retrograde filling of the aneurysmal sac. This result allowed us to accomplish an occlusion of the whole aneurysm without the coiling technology/ stenting. Selective catheterization of the aneurysmal sac with a microcatheter Echelon 14 was done, which resulted in progressive occlusion of the distal to proximal part. Control angiography at the end of the procedure confirmed the complete exclusion from the circulation at the level of the aneurismal sac, and good retrograde filling of the leptomeningeal collateral by the occipital territory, especially by branches coming from the right carotid circulation.

\section{Case 2}

A 49-year-old female patient presented with an acute onset of headache with meningeal irritation signs. At the time of admission, her Glasgow Coma Scale score was 13. She had no motor or sensory deficits. CT of the brain was done and showed a subarachnoid hemorrhage with extension to the basal cistern, fourth and 
Wael Osman et al.: Optional Endovascular Therapy of Dissecting Posterior Cerebral Artery Aneurysm
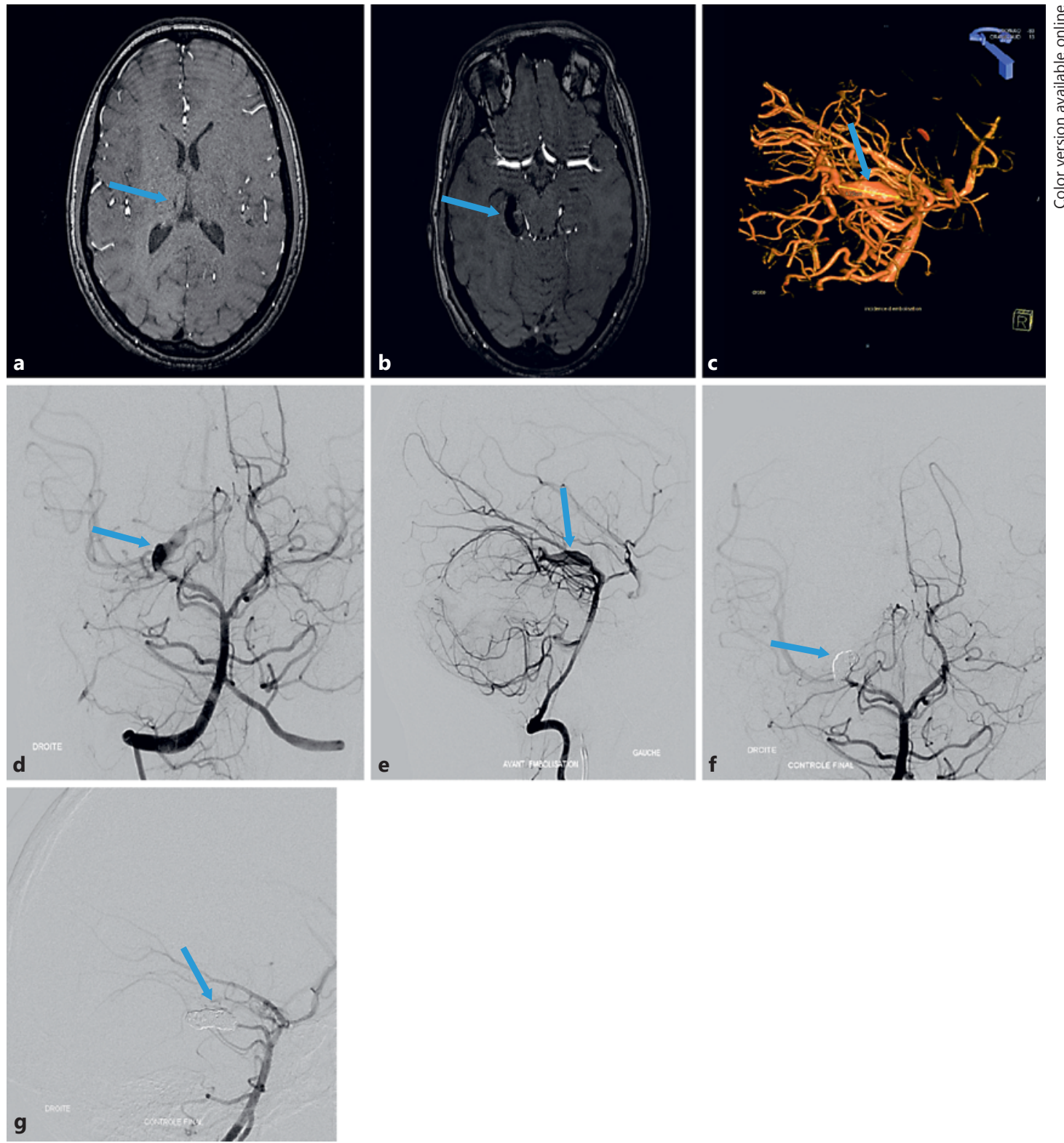

Fig. 1. A 19-year-old female patient presented with recurrent stroke. An MRI scan of the brain was done before treatment. 3D DSA before treatment and DSA after treatment. a MRI of the brain showing a right thalamic hypointense area. $\mathbf{b}$ MRI of the brain showing a right perimesencephalic hypointense area. c 3D DSA, lateral view, showing a fusiform dissection aneurysm in the right posterior cerebral artery of the P2-P3 segment, measuring $12 \mathrm{~mm}$ in length and 4.5-6 $\mathrm{mm}$ in height. $\mathbf{d}$ DSA, antero-posterior view, showing right PCA dissecting aneurysm before endovascular therapy. e DSA, lateral view, showing right PCA dissecting aneurysm before endovascular therapy. f DSA, antero-posterior view, showing right PCA dissecting aneurysm after complete occlusion. g DSA, lateral view, showing right PCA dissecting aneurysm after complete occlusion.

third ventricles, grade 4 on the Fisher scale and grade 3 on the Hunt and Hess scale. There was no history of trauma, hypertension, diabetes or other diseases. The patient underwent diagnostic DSA immediately after admission and revealed negative results. Angiography was repeated after 2 weeks, which showed the presence of dissecting aneurysm of the right PCA at the P2 segment, measuring $7 \times 6 \mathrm{~mm}$ with neck $4 \mathrm{~mm}$. The patient underwent the embolization reconstruction technique (coiling and stenting) in two stages due to incomplete occlusion in the first stage, with no neurological complications after embolization (Fig. 2). 

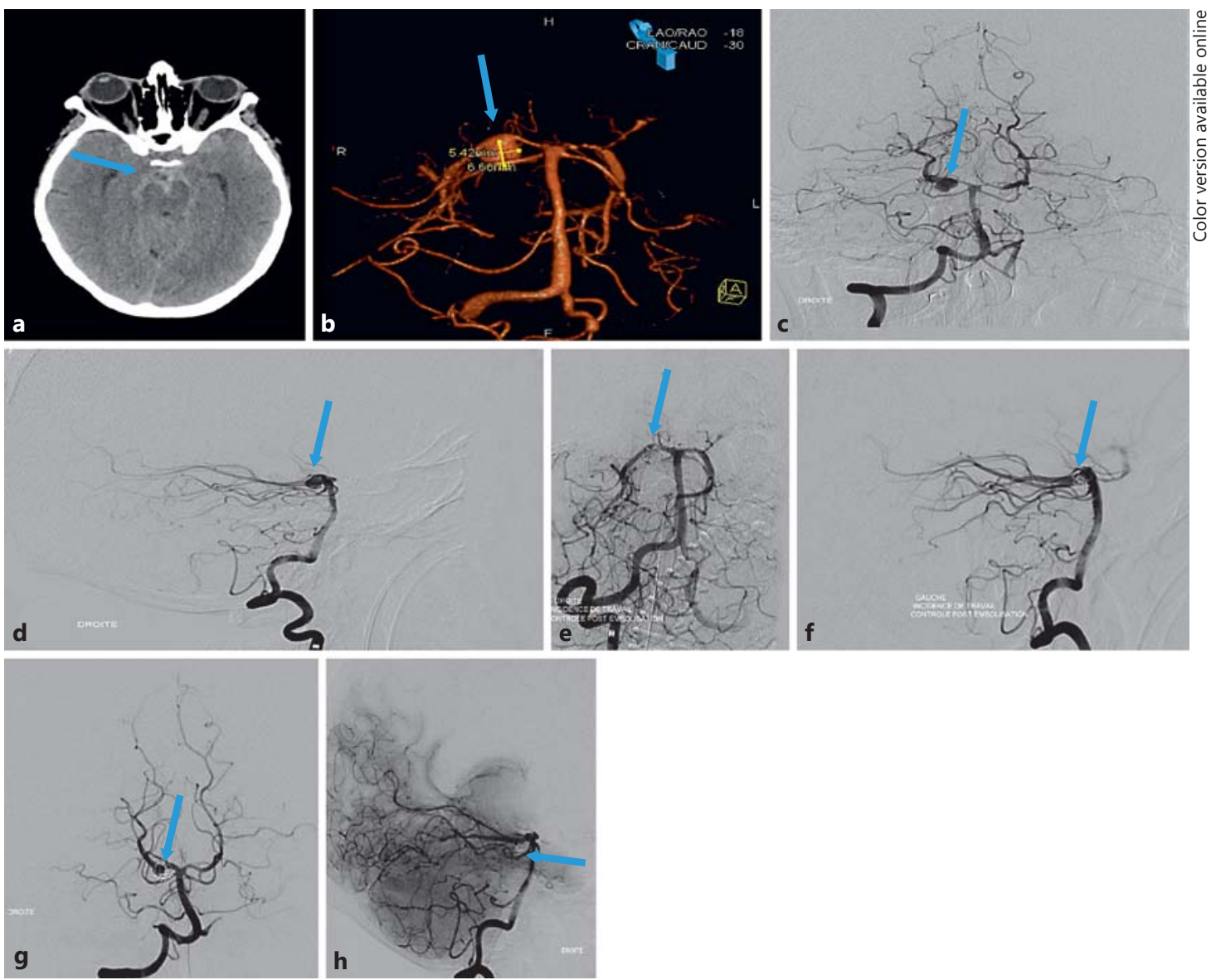

Fig. 2. A 49-year-old female patient presented with an acute onset of headache with meningeal irritation signs. CT of the brain was performed before treatment; DSA was done before treatment and after embolization. a CT of the brain showing a subarachnoid hemorrhage with extension to the basal cistern. b 3D DSA, antero-posterior view, showing right posterior cerebral artery aneurysm at the P2 segment, measuring $7 \times$ $6 \mathrm{~mm}$ with a 4-mm neck. c DSA, antero-posterior view, showing right PCA aneurysm. d DSA, lateral view, showing right PCA aneurysm. e DSA, antero-posterior view, showing right PCA aneurysm following embolization. g DSA, lateral view, showing right PCA aneurysm following embolization. $\mathbf{f}$ DSA, lateral view, showing right PCA poststent placement. g DSA, antero-posterior view, showing right PCA poststent placement (Enterprise stent $4.5 \times 14$, to cover the whole segment the second Solitary stent $4 \times 15$ was deployed inside the Enterprise stent). $\mathbf{h}$ DSA, lateral view, showing right PCA poststent placement and final control.

\section{Technique}

First Session

The operation was done under general anesthesia, with bifemoral puncture, using a 6-Fr femoral sheath at the level of the right and left femoral artery. Selective catheterization of the right vertebral artery with a 6-Fr Fargomax catheter, and selective catheterization of the left vertebral artery by a 5-Fr Envoy catheter, followed by injection of the right and left vertebral artery were done. The plan was to apply the reconstruction technique using coils plus a stent. We used a Prowler Select Plus microcatheter to access the left vertebral artery, while the access to the aneurysm was achieved via the microcatheter Echelon 10; the Enterprise stent $4.5 \times 22 \mathrm{~mm}$ was delivered with coils (4 coils microplex 10; $6 \times 15 \mathrm{~cm}$ [lot 10050613 ref 100615 CC-V], $2 \times 6 \mathrm{~cm}$ [lot 10070813 ref $100206 \mathrm{HS}-\mathrm{V}$ ], $2 \times 2 \mathrm{~cm}$ [lot 10072113 ref $100202 \mathrm{HS}-\mathrm{V}-$ lot 10061513 ref 100202 HC-S-V] [×2]).

The result was incomplete occlusion of the right posterior cerebral artery aneurysm and a second session was needed. 
Second Session 5 Months Later

The operation was done under general anesthesia, with bifemoral puncture, and a 6-Fr femoral sheath at the level of the right and left femoral artery. Selective catheterization of the right vertebral artery and selective catheterization of the right internal carotid artery at the same time by a 6-Fr catheter Chaperon were done, followed by an antero-posterior, lateral and 3D view at the level of the vertebral artery. We made an occlusion at the level of the basilar artery to show the collateral circulation of the right internal carotid artery. Angiography was done and detected a right posterior cerebral aneurysm at the level of the P2 segment measuring $5 \mathrm{~mm}$ in its big axis $\times 2.5$. The occlusion test of the bearing vessel was negative. Selective catheterization of the P2 segment with the aid of the microcatheter Proler Select Plus 21 was done, then the Enterprise stent $4.5 \times 14$ was deployed. To cover the whole segment we used the second Solitary stent $4 \times 15$ inside the Enterprise stent. Finally, control angiography was done to show permeability of the two stents (Fig. 2).

\section{Case 3}

A 45-year-old female patient presented with left-side sensory manifestation and left facial pain starting from teeth to the ear, with no history of trauma, smoking, headache, hypertension, diabetes or other diseases. The patient sought medical advice, and MRI and MRA were performed and showed hypovolemia of the corpus callosum and right PCA aneurysm. Also, DSA was done in the antero-posterior, lateral and 3D view. We found a right posterior cerebral aneurysm at the level of the P2 segment. This aneurysm measured $6 \mathrm{~mm}$ in its big axis and $3.5 \mathrm{~mm}$ in its transverse axis, with a wide neck of $5 \mathrm{~mm}$. Endovascular therapy was recommended to the patient. The operation was done and complete occlusion of the right PCA aneurysm was achieved without residual neck or neurological complications. Follow-up MRA was done and showed no residual neck of aneurysm and complete occlusion (Fig. 3).

\section{Technique}

The operation was performed under general anesthesia. Right femoral puncture with a 6-Fr sheath was done, followed by selective catheterization of the left vertebral artery with a 6-Fr GUIDE ENVOY catheter, followed by injection of VISIPAQUE 320 (150 mL).

The angiography was done in the anteroposterior, lateral and 3D view. We found a right posterior cerebral aneurysm at the level of the P2 segment. This aneurysm measures $6 \mathrm{~mm}$ in its big axis and $3.5 \mathrm{~mm}$ in its transverse axis, with a wide neck of $5 \mathrm{~mm}$. Installation of a HYPERGLIDE $4 \times 15 \mathrm{~mm}$ balloon at the neck of the aneurysm was done, followed by selective catheterization of the aneurysmal neck with a microcatheter Echelon 10. Delivery of 5 coils was done by using balloon remodeling technique (3D EV3 AXIUM $6 \mathrm{~mm} \times 20$ cm, REF QC-6-20-3D LOT 8075360, HELIX EV3 AXIUM 3 mm $\times 4$ cm, REF QC-3-4-HELIX LOT 8075297 [×2], HELIX EV3 HELIX $2 \mathrm{~mm} \times 3 \mathrm{~cm}$, QC-2-3-HELIX LOT 8075284 [×2]). Finally, we made a satisfactory occlusion. The final control showed a good-quality occlusion of the aneurismal sac with the permeability of the PCA at the level of segments P2, P3, and P4.

\section{Discussion}

Dissecting PCA aneurysm was based on the angiographic appearance described as "pearl and string" or "blow out." PCA is divided into the proximal segment and the distal segment by the confluence of the posterior communicating artery [8]. Aneurysms of the PCA are rare. The PCA supplies part of the temporal cortex, the calcarine and the occipital cortex as well as parts of the brain stem and thalamus. PCA aneurysms may be saccular, fusiform, or dissecting and can be located at various segments of the PCA. Dissecting aneurysms may occlude the PCA causing homonymous hemianopia if collateral circulation is not sufficient [1]. Endovascular treatment consists of selective occlusion of the aneurysm or occlusion of the parent artery. Surgery of PCA aneurysms is technically challenging owing to the complexity of the perforating branches from the PCA and their relationship with cranial nerves and the upper brain stem [9]. 
The PCA can anatomically be subdivided into 4 segments [10]. The P1 segment extends from the tip of the basilar artery to the origin of the posterior communicating artery. The occulomotor nerve has an intimate relationship with the P1 and courses between the P1 and the proximal superior cerebellar artery. The P2 segment extends from the posterior communicating artery to the dorsal aspect of the midbrain. From the P1 and P2 segment, perforating arteries to the thalamus (thalamo-perforating and posterior choroidal arteries) and brain stem (pedunculo-perforating and long circumflex arteries) arise. The P3 and P4 segments extend distally from the dorsal aspect of the midbrain and supply the calcarine and parieto-
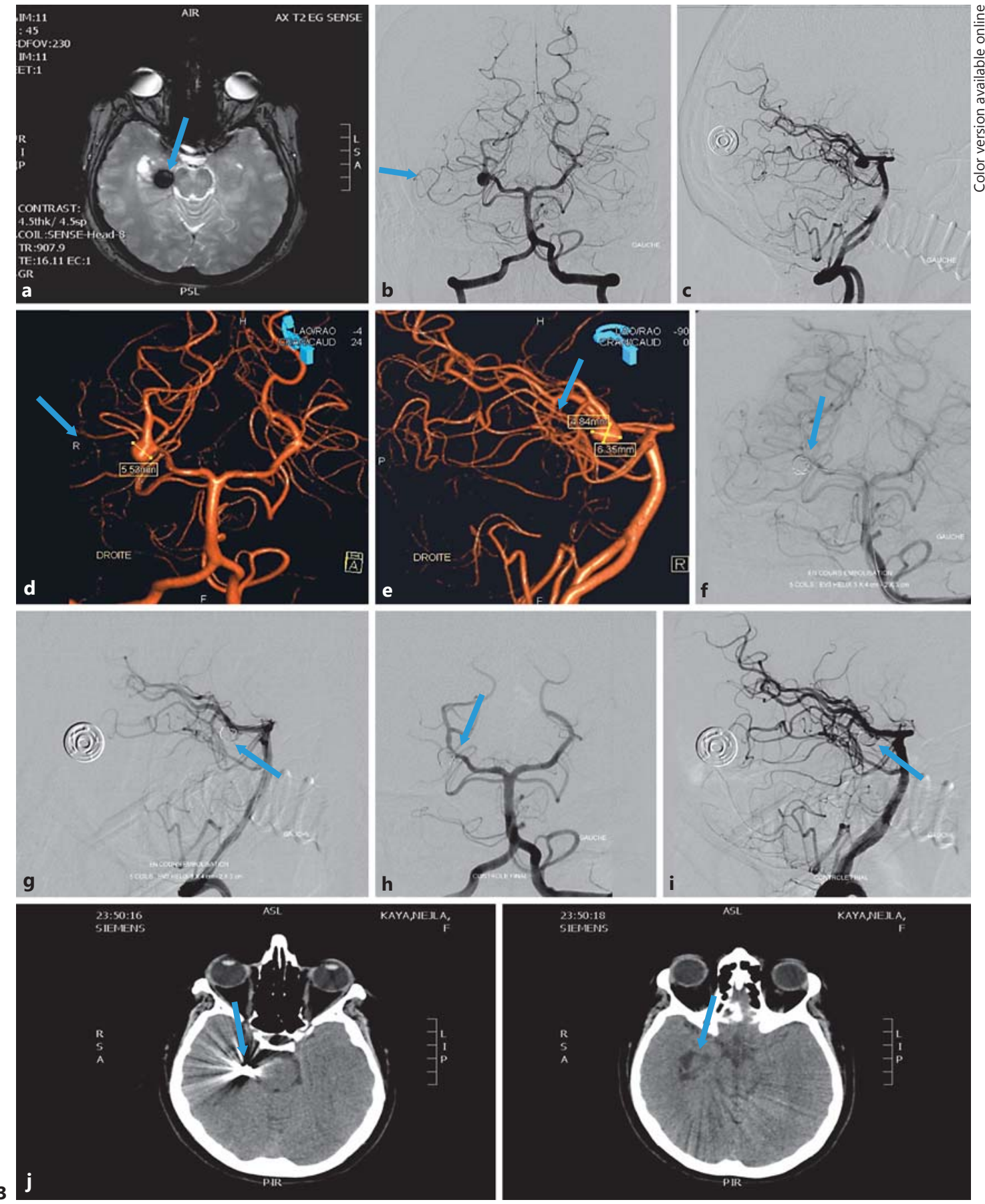

(For legend see next page.) 
occipital cortex. A rich collateral network exists between both the deep and superficial territories of the PCA and other arteries which include the following:

1. Collateral circulation between the lateral posterior choroidal (branch of the P2 segment) and the anterior choroidal arteries.

2. Collateral circulation between the long circumflex arteries (branches of the P1 segment) and the superior cerebellar artery territory at the level of the quadrigeminal plate.

3. Collateral circulation between the splenial artery (branch of the P3-P4 segments) and the posterior pericallosal artery (branch of the anterior cerebral artery).

4. Collateral circulation between the inferior temporal branches of the PCA and the superior temporal branches of the middle cerebral artery [11].

Our patients were young; our first case was 19 years old, the second was aged 49 years and the third one 45 years, which is younger than the average age of patients with aneurysms occurring at other anatomic sites: Ferrante et al. [1] reported of patients being 50-60 years old, while in the study of Luo et al. [12], the age of patients was 18-57 years. All patients were female (100\%). Fullerton et al. [2] and Kawahara et al. [13] reported that PCA dissections were predominant in females, with a female-male ratio of 3.1:1. Also, Luo et al. [12] found a female-male ratio of 2:1. This may be due to hormonal effects. Our patients have different morphologic features (the first one was fusiform, while the second and third were saccular) and presentation. One of our patients presented with headache, subarachnoid hemorrhage, and a history of migraine, while the other 2 patients presented with cerebral infarction. This finding was congruent with the observation by Kawahara et al. [13], who found that the most common symptom of PCA dissection is headache, predominantly in the occipital and posterior cervical regions. Dissecting intracranial aneurysms usually present with large cerebral infarcts but may also present with subarachnoid hemorrhage or both. They may be associated with severe neurological deficits, often leading to death. Isolated PCA dissections tend to present with ischemic symptoms and have a more benign clinical course and prognosis [13]. Taqi et al. [14] found that subarachnoid hemorrhage is present in approximately $86 \%$ of cases (6 of 7), and 14\% ( 1 of 7) have ischemic stroke. In other study, $54 \%$ had subarachnoid hemorrhage, $25 \%$ focal neurological deficits without subarachnoid hemorrhage, and $21 \%$ were discovered accidentally [3,11, 15-26].

As regards the possible cause, we found that the first patient, who presented with cerebral infarction, was a smoker and was taking the contraceptive pill, the second patient presented with subarachnoid hemorrhage and had a history of migraine, while the third one presented with cerebral infarction with no possible cause detected. Sherman et al. [27] found that the possible cause in 2 patients of their study is trauma as the onset of the symptoms was shortly after a minor head trauma without loss of consciousness. Hamada et al. [18] noted various

Fig. 3. A 45-year-old female patient presented with left-side sensory manifestation and left facial pain. T2 MRI of the brain was performed, DSA before embolization, during embolization, after embolization and CT of the brain after endovascular therapy. a T2 MRI of the brain showing a right perimesencephalic hypointense area. b DSA, antero-posterior view, showing right PCA aneurysm before endovascular therapy. c DSA, lateral view, showing right PCA aneurysm before endovascular therapy. d DSA, 3D antero-posterior view, showing right PCA aneurysm at the level of the P2 segment. This aneurysm measures $6 \mathrm{~mm}$ in its big axis, 3.5 mm in its transverse axis with a wide 5-mm neck. e DSA, 3D lateral view, right PCA at the level of the P2 segment. This aneurysm measures $6 \mathrm{~mm}$ in its big axis, $3.5 \mathrm{~mm}$ in its transverse axis with a wide 5-mm neck. f DSA, antero-posterior view, showing right PCA aneurysm during endovascular therapy with complete occlusion of aneurysm. g DSA, lateral view, showing right PCA aneurysm during endovascular therapy with complete occlusion of aneurysm. $\mathbf{h}$ DSA, antero-posterior view, showing right PCA aneurysm after endovascular therapy with complete occlusion of aneurysm. i DSA, lateral view, showing right PCA aneurysm after endovascular therapy with complete occlusion of aneurysm. $\mathbf{j}$ CT of the brain after treatment. 
etiologies including hypertension, trauma, smoking, infection (mycotic, syphilis), migraine, fibromuscular dysplasia, homocystinuria, and mixed connective tissue diseases. Isolated PCA dissections most commonly occur near the P1-P2 junction, which is close to the free border of the tentorium cerebelli. It has been suggested that traumatic aneurysms, including dissections, may occur in mild head trauma secondary to a stretch or shear injury of the vessels [17]. This finding of Lazinski et al. [17] agrees with that of Drake et al. [9], Taqi et al. [14], and our finding. While Hamada et al. [18] said that arterial hypertension has commonly been associated with extracranial carotid dissection, few patients with posterior circulation dissection are found to be hypertensive. Thus, we suggest making a large-scale survey of dissecting PCA to define the etiology.

As regards treatment, we treated 3 cases with different methods of endovascular therapy: in the first one, we sacrificed the parent right PCA after a positive balloon occlusion test for good collateral circulation from the right internal carotid that fills the aneurysm after occlusion without postoperative complications. This optional treatment was used by Hallacq et al. [26], Hanel et al. [28], as well as Coert et al. [29].

Taqi et al. [14] treated 4 cases out of 7 (57\%), Lazinski et al. [17] treated 2 cases out of 6 patients, Oran et al. [23] treated 4 cases, and Lv et al. [22] treated 8 out of 8 patients without complications. Luo et al. [12] studied 10 patients with PCA aneurysms; 6 of them were dissected (1 at the P2-P3 segment, 3 cases at the P3, and 2 at the P2 segment). All of them had coil embolization and occlusion of the parent artery as the collateral circulations distal to the PCA are quite rich, such as the cortical branch of the other PCA, branches of the posterior choroid artery, branches of the middle and anterior cerebral arteries, and branches of the circumflex arteries. Blood supply of the distal brain tissue can be compensated by the opening of the collateral circulations.

The second patient was treated in two sessions. In the first session, we deployed the neuroform stent and coiling with incomplete occlusion. The patient was given intra-arterial abciximab during the procedure. A few months later, we applied a solitaire stent with satisfactory occlusion. The problem of this method was that the patient had to be administered an antiplatelet (clopidogrel) before the deployment of the stent. This is not allowed in patients with hemorrhage, as it may increase the risk of hemorrhage, rupture or extension of the lesion; therefore, a balance must be found between risk and benefit for the patient $[2,25]$. Taqi et al. [14] performed stent-assisted coiling in 3 out of 7 patients (one of them developed new sac formation and required parent artery occlusion to be sacrificed distally to the thalamic perforator without a new neurological deficit). While the other 2 patients had no residual, all 3 patients were given preoperative and intraoperative antiplatelet drugs. One of the patients developed asymptomatic thrombus and was successfully treated with intraarterial abciximab during the procedure.

In the third patient, the aneurysm was occluded by coils without sacrificing the parent artery, and without residual or postoperative complications. Also, Taqi et al. [14] treated 1 patient with coiling alone without occluding the artery and had good results at the end of the procedure.

Some authors suggested no treatment in patients who are asymptomatic or have resolved neurological deficits. Hypertensive patients must be controlled to prevent progression of dissection and possible rebleeding or worsened ischemia $[25,30]$, while patients with ruptured aneurysm with subarachnoid hemorrhage or progressive neurological deficits require surgical or endovascular therapy [17].

However, anticoagulation is recommended for patients with dissecting aneurysm presenting with ischemic stroke [17]. Sherman et al. [27] chose conservative antiplatelet therapy in 2 patients. Also, Pozzati et al. [16] reported 2 cases of nonhemorrhagic PCA dissecting aneurysm that resolved spontaneously without anticoagulation. 


\section{Conclusion}

Dissecting PCA aneurysm is a rare type of aneurysm, of unknown etiology, of different clinical presentations, and more common in females. There are different endovascular procedures for treatment such as coiling and sacrificing the parent artery, stent-assisted coils and coiling without sacrificing the parent artery. In the future, we suggest the application of these procedures to a large number of patients to identify the safest and most effective method for treatment, and the natural history of dissecting PCA aneurysm.

\section{Disclosure Statement}

The authors have nothing to disclose.

\section{References}

1 Ferrante L, Acqui M, Trillo G, Lunardi P, Fortuna A: Aneurysms of the posterior cerebral artery: do they present specific characteristics? Acta Neurochir (Wien) 1996;138:840-852.

2 Fullerton HJ, Johnston SC, Smith WS: Arterial dissection and stroke in children. Neurology 2001;57:11551160.

3 Kitazawa K, Tanaka Y, Muraoka S, Okudera H, Orz Y, Kyoshima K, Kobayashi S: Specific characteristics and management strategies of cerebral artery aneurysms: report of eleven cases. J Clin Neurosci 2001;8:23-26.

4 Taylor CL, Kopitnik TA Jr, Samson DS, Purdy PD: Treatment and outcome in 30 patients with posterior cerebral artery aneurysms. J Neurosurg 2003;99:15-22.

5 Honda M, Tsutsumi K, Yokoyama H, Yonekura M, Nagata I: Aneurysms of the posterior cerebral artery: retrospective review of surgical treatment. Neurol Med Chir (Tokyo) 2004;44:164-168; discussion 169.

6 Ng PY, Yeo TT: Petrosal approach for a large right posterior cerebral artery (P2) aneurysm. J Clin Neurosci 2000;7:445-446.

7 van Rooij WJ, Sluzewski M, Beute GN: Endovascular treatment of posterior cerebral artery aneurysms. AJNR Am J Neuroradiol 2006;27:300-305.

8 Milisavljevic M, Marinkovic S, Marinkovic Z, Malobabic S: Anatomic basis for surgical approach to the distal segment of the posterior cerebral artery. Surg Radiol Anat 1988;10:259-266.

9 Drake CG, Peerless SJ, Hernesniemi J: Surgery of Vertebrobasilar Aneurysms: London, Ontario Experience on 1,767 Patients. New York, Springer Science \& Business Media, 2012.

10 Zeal AA, Rhoton AL Jr: Microsurgical anatomy of the posterior cerebral artery. J Neurosurg 1978;48:534-559.

11 Ciceri EF, Klucznik RP, Grossman RG, Rose JE, Mawad ME: Aneurysms of the posterior cerebral artery: classification and endovascular treatment. AJNR Am J Neuroradiol 2001;22:27-34.

12 Luo Q, Wang $\mathrm{H}, \mathrm{Xu} \mathrm{K}, \mathrm{Yu}$ J: Endovascular treatments for distal posterior cerebral artery aneurysms. Turk Neurosurg 2012;22:141-147.

13 Kawahara I, Hiu T, Onizuka M, Toda K, Baba H, Yonekura M: Isolated posterior cerebral artery dissection - case report and review of the literature. No Shinkei Geka 2003;31:671-675.

14 Taqi MA, Lazzaro MA, Pandya DJ, Badruddin A, Zaidat O0: Dissecting aneurysms of posterior cerebral artery: clinical presentation, angiographic findings, treatment, and outcome. Front Neurol 2011;2:38.

15 Berger MS, Wilson CB: Intracranial dissecting aneurysms of the posterior circulation. Report of six cases and review of the literature. J Neurosurg 1984;61:882-894.

16 Pozzati E, Padovani R, Fabrizi A, Sabattini L, Gaist G: Benign arterial dissections of the posterior circulation. J Neurosurg 1991;75:69-72.

17 Lazinski D, Willinsky RA, TerBrugge K, Montanera W: Dissecting aneurysms of the posterior cerebral artery: angioarchitecture and a review of the literature. Neuroradiology 2000;42:128-133.

18 Hamada J, Morioka M, Yano S, Todaka T, Kai Y, Kuratsu J: Clinical features of aneurysms of the posterior cerebral artery: a 15-year experience with 21 cases. Neurosurgery 2005;56:662-670; discussion 662-670.

19 Vilela P, Goulao A: Paediatric dissecting posterior cerebral aneurysms: report of two cases and review of the literature. Neuroradiology 2006;48:541-548.

20 Nistri M, Perrini P, Di Lorenzo N, Cellerini M, Villari N, Mascalchi M: Third-nerve palsy heralding dissecting aneurysm of posterior cerebral artery: digital subtraction angiography and magnetic resonance appearance. J Neurol Neurosurg Psychiatry 2007;78:197-198.

21 Renard D, Milhaud D: Dissecting aneurysm of the posterior cerebral artery. N Engl J Med 2007;357:e27.

22 Lv X, Li Y, Jiang C, Yang X, Wu Z: Parent vessel occlusion for P2 dissecting aneurysms of the posterior cerebral artery. Surg Neurol 2009;71:319-325; discussion 325. 
23 Oran I, Cinar C, Yagci B, Tarhan S, Kiroglu Y, Serter S: Ruptured dissecting aneurysms arising from nonvertebral arteries of the posterior circulation: endovascular treatment perspective. Diagn Interv Radiol 2009; 15:159-165.

24 Chang SW, Abla AA, Kakarla UK, Sauvageau E, Dashti SR, Nakaji P, Spetzler RF: Treatment of distal posterior cerebral artery aneurysms: a critical appraisal of the occipital artery-to-posterior cerebral artery bypass. Neurosurgery 2010;67:16-25; discussion 25-26.

25 Maillo A, Diaz P, Morales F: Dissecting aneurysm of the posterior cerebral artery: spontaneous resolution. Neurosurgery 1991;29:291-294.

26 Hallacq P, Piotin M, Moret J: Endovascular occlusion of the posterior cerebral artery for the treatment of p2 segment aneurysms: retrospective review of a 10-year series. AJNR Am J Neuroradiol 2002;23:1128-1136.

27 Sherman P, Oka M, Aldrich E, Jordan L, Gailloud P: Isolated posterior cerebral artery dissection: report of three cases. AJNR Am J Neuroradiol 2006;27:648-652.

28 Hanel RA, Boulos AS, Sauvageau EG, Levy EI, Guterman LR, Hopkins LN: Stent placement for the treatment of nonsaccular aneurysms of the vertebrobasilar system. Neurosurg Focus 2005;18:E8.

29 Coert BA, Chang SD, Do HM, Marks MP, Steinberg GK: Surgical and endovascular management of symptomatic posterior circulation fusiform aneurysms. J Neurosurg 2007;106:855-865.

30 Sasaki O, Koizumi T, Ito Y, Sorimachi T, Koike T, Tanaka R: Dissecting aneurysm of the posterior cerebral artery treated with proximal ligation. Surg Neurol 1992;37:394-401. 